EPJ Web of Conferences 41, 01013 (2013)

DOI: $10.1051 /$ epjconf/20134101013

C) Owned by the authors, published by EDP Sciences, 2013

\title{
Quasi-phase-matching of high harmonic generation using counter-propagating pulses
}

\author{
K. O’ Keeffe ${ }^{1}$, and S.M. Hooker ${ }^{1}$ \\ ${ }^{1}$ Department of Physics, University of Oxford, Clarendon Laboratory, Parks Road, OX1 3PU, United \\ Kingdom,
}

\begin{abstract}
We investigate quasi-phase-matching over a range of harmonic orders using trains of up to 8 uniformly-spaced counter-propagating pulses. For trains of up to 4 pulses the measured enhancement of the harmonic signal scales with the number of pulses $\mathrm{N}$ as $(\mathrm{N}+1)^{2}$, as expected. However, for trains with $\mathrm{N}>4$, no further enhancement of the harmonic signal is observed. The effect is ascribed to changes in the coherence length with the generating medium. The pressure dependence of quasiphase-matching is also investigated and it is found that additional peaks in the harmonic intensity are consistent with higher-order QPM processes.
\end{abstract}

\section{Introduction}

High harmonic generation (HHG) offers a straightforward way for generating coherent soft xray radiation in the XUV region. However, a significant drawback of HHG is that it is inefficient due to the difference in the phase velocities of the driving and generated radiation. One way to overcome this problem, and thereby greatly increase the efficiency of HHG, is to employ quasiphase-matching (QPM). In this approach, HHG is suppressed in regions where the locally generated radiation is out of phase with the harmonic beam. Radiation generated in the remaining regions combines constructively, resulting in the monotonic growth of the harmonic intensity. QPM can be achieved using a train of counter-propagating laser pulses [1]; suppressing harmonic generation in those regions in which the driving laser pulse overlaps with a counter-propagating pulse. By matching the width, w, and separation, $d$, of the counterpropagating pulses to the coherence length of the generated harmonics it is possible to suppress HHG in multiple out-of-phase zones. In the case of QPM using counter-propagating pulses the enhancement of the harmonic signal is expected to scale as $(\mathrm{N}+1)^{2}$, where $\mathrm{N}$ is the number of pulses in the train. Here we investigate QPM by trains of up to 8 uniformly-spaced laser pulses colliding with a driving laser pulse within a hollow capillary waveguide [2].

\section{QPM with uniformly-spaced pulses}

The output from a $4 \mathrm{~mJ}$, $50 \mathrm{fs}$ laser system was split to produce a driver and counterpropagating pulse. The energy of the driver was $500 \mu \mathrm{J}$ and was used to generate high harmonics in a low density Ar gas contained in a $4 \mathrm{~cm}$ long, hollow-core waveguide with an inner diameter of $102 \mu \mathrm{m}$. The pulse train was generated by sending the counter-propagating

This is an Open Access article distributed under the terms of the Creative Commons Attribution License 2.0, which permits unrestricted use, distribution, and reproduction in any medium, provided the original work is properly cited. 
pulse through a sequence of P birefringent plates [4]. This arrangement generates a train of up to $2^{\mathrm{P}}$ uniformly-spaced pulses, and the number of pulses and width of each pulse in the train can be varied by changing the configuration of the crystal array, allowing different coherence lengths to be matched. The driver and counter-propagating beams were coupled into opposite ends of the hollow-core waveguide and their temporal overlap adjusted so that they collided inside the waveguide.

Experiments were performed to observe QPM for 2 different pulse train configurations: a 2pulse train with $\mathrm{d}=\mathrm{w}=1080 \mu \mathrm{m}$ and a 4-pulse train with $\mathrm{d}=\mathrm{w}=540 \mu \mathrm{m}$. The peak intensities of the pulses were the same in both cases. QPM was observed over a range of harmonic orders as shown in Figure 1(a). For the 4-pulse train strong enhancement of harmonic orders $q=23$ - 33 was observed, with the strongest enhancement of $40 \pm 5$ being observed for $q=27$. For the experiments presented here the maximum harmonic order which could be generated was $\mathrm{q}=$ 35. The good agreement between the measured enhancements and the calculated enhancement shown in Figure 1(a) suggests that QPM could be extended to higher order harmonics by employing a suitable pulse train.

Experiments were then performed to investigate the effect of changing the number of pulses in a train with $\mathrm{d}=\mathrm{w}=540 \mathrm{~mm}$. Trains of $1,2,4$, or 8 pulses could be obtained by rotating the appropriate birefringent plates in the array. In each case the intensity per counter-propagating pulse was kept constant. Figure 1 (b) shows the measured enhancement of the $27^{\text {th }}$ harmonic as a function of $\mathrm{N}$, together with the expected $(\mathrm{N}+1)^{2}$ scaling. The measured enhancement scales as expected up to $\mathrm{N}=4$. However, for trains of $\mathrm{N}=8$ pulses the enhancement is observed to be no greater than that measured for 4 pulses. This observation may be explained by considering the effect of variations of the coherence length within the waveguide. It has previously been shown that the coherence length of harmonics generated in a hollow-core waveguide varies as a function of longitudinal position owing to variation of the intensity of the driving laser $[4,5]$. Simulations have shown that a variation in the coherence length of $10 \%$ across the generating region is sufficient to result in a maximum enhancement of 35 for a train of 8 uniformly spaced pulses, instead of the expected enhancement of 81 [2]. In order to extend the number of pulses which can be used, and hence increase the harmonic output, it will be necessary to overcome the problem of a varying coherence length. One possibility would be to employ pulse trains in which the pulse spacing can be varied with the train [6].
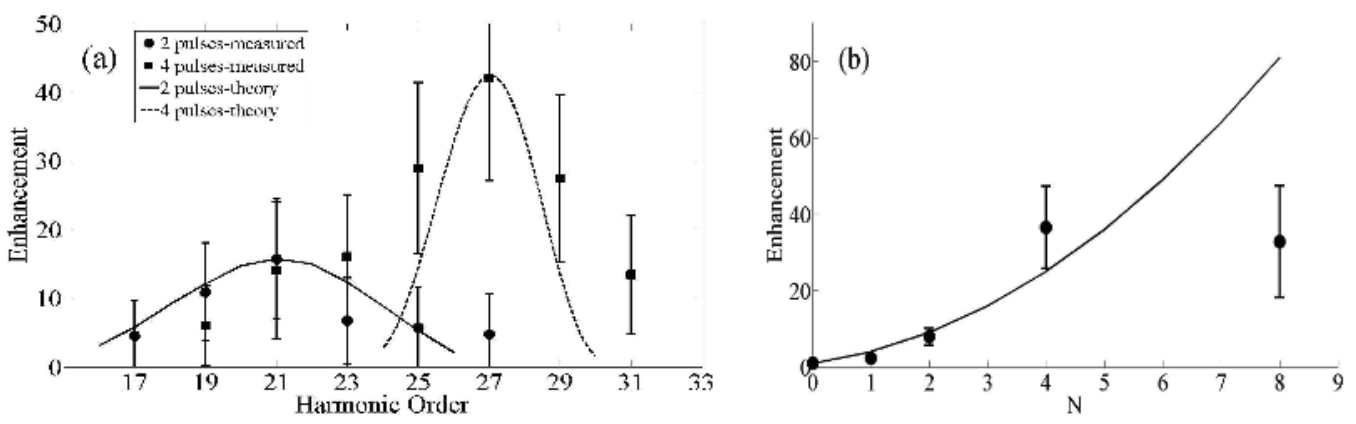

Fig. 1. (a) Measured enhancement of the harmonic signal for two separate pulse train configurations, corresponding to 2 pulses of $1080 \mathrm{~mm}$ width (circles) and 4 pulses of $540 \mathrm{~mm}$ width (squares). The expected enhancement for 2 pulses (solid line) and 4 pulses (dashed line) is also illustrated. (b) Measured enhancement of the 27th harmonic as a function of number of pulses in the counterpropagating train (circles), as well as the expected scaling of harmonic intensity with pulse number (solid line). 


\section{Pressure dependence of QPM}

The dependence of QPM on pressure was investigated using a train of 4 pulses with $\mathrm{w}=\mathrm{d}=$ $540 \mu \mathrm{m}$, and the position of the timing slide adjusted to give a maximum enhancement of the 27 th harmonic at a pressure of $11 \mathrm{mbar}$ of argon. The pressure in the waveguide was then varied from 0 to $50 \mathrm{mbar}$ in steps of $2 \mathrm{mbar}$, and in steps of $10 \mathrm{mbar}$ from 60 to $150 \mathrm{mbar}$. At each pressure the harmonic spectrum was recorded with and without the pulse train. Figure 2 shows the recorded harmonic signals as a function of pressure for $q=27$. The peak observed near 130 mbar, both with and without the pulse train, arises from true phase-matching. This is confirmed by calculating the harmonic intensity for an ionization fraction of $\eta=0.026$, which corresponds to $\Delta \mathrm{k}=0$ at $130 \mathrm{mbar}$ for $\mathrm{q}=27$. In the presence of a pulse train two additional peaks are also observed at approximately 14 and 40mbar: these correspond to first-order $(\mathrm{m}=1)$ and third-order $(\mathrm{m}=3) \mathrm{QPM}$, respectively. The observed optimum pressures for first and third-order QPM are calculated to occur for ionization fractions of $\eta=0.125$ and $\eta=0.25$, respectively. This variation of the ionization fraction with pressure may be caused by local changes of the driver intensity in the waveguide.

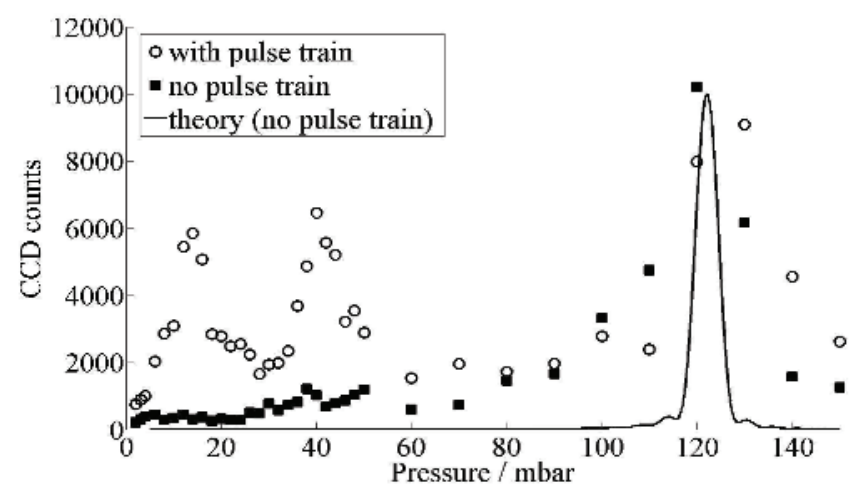

Fig. 2. Measured harmonic signal of $q=27$ as a function of pressure with (open circles), and without (filled squares) a counter-propagating train of $\mathrm{N}=4$ pulses. The solid line shows the calculated intensity of $\mathrm{q}=27$, in the absence of a pulse train. The peak around $130 \mathrm{mbar}$ corresponds to true-phase matching, while the peaks at $14 \mathrm{mbar}$ and $40 \mathrm{mbar}$ are the result of first and third-order QPM, respectively.

\section{References}

1. X. Zhang et al., Nat. Phys. 14, 270 (2007)

2. K. O'Keeffe et al., Opt. Exp. 20, 6236, (2012)

3. B. Dromey et al., Appl. Opt., 46, 5142, (2007)

4. T. Robinson et al. J Opt. Soc. Am. B, 27, 763, (2010)

5. A. Lytle et al., Phys, Rev Lett, 98, 123904, (2007)

6. K. O'Keeffe et al., J. Opt., 12, 015201, (2010) 